\title{
The effect of histological subtypes on survival outcome in nasopharyngeal carcinoma after extensive follow up
}

\author{
San-Gang Wu ${ }^{1 \#}$, Chen-Lu Lian ${ }^{1 \#}$, Jun Wang ${ }^{1}$, Wen-Wen Zhang ${ }^{2}$, Jia-Yuan Sun ${ }^{2}$, Qin Lin ${ }^{1}$, Zhen-Yu He ${ }^{2}$ \\ ${ }^{1}$ Department of Radiation Oncology, Cancer Hospital, the First Affiliated Hospital of Xiamen University, Teaching Hospital of Fujian Medical \\ University, Xiamen 361003, China; ${ }^{2}$ Department of Radiation Oncology, Sun Yat-sen University Cancer Center, State Key Laboratory of Oncology \\ in South China, Collaborative Innovation Center of Cancer Medicine, Guangdong Key Laboratory of Nasopharyngeal Carcinoma Diagnosis and \\ Therapy, Guangzhou 510060, China \\ Contributions: (I) Conception and design: SG Wu, CL Lian, Q Lin, ZY He; (II) Administrative support: SG Wu, CL Lian; (III) Provision of study \\ materials or patients: SG Wu; (IV) Collection and assembly of data: SG Wu, ZY He; (V) Data analysis and interpretation: J Wang, WW Zhang, JY \\ Sun; (VI) Manuscript writing: All authors; (VII) Final approval of manuscript: All authors. \\ "These authors contributed equally to this work. \\ Correspondence to: Qin Lin. Department of Radiation Oncology, Cancer Hospital, the First Affiliated Hospital of Xiamen University, Teaching \\ Hospital of Fujian Medical University, Xiamen 361003, China. Email: linqin9531@126.com; Zhen-Yu He. Department of Radiation Oncology, \\ Sun Yat-sen University Cancer Center, State Key Laboratory of Oncology in South China, Collaborative Innovation Center of Cancer Medicine, \\ Guangdong Key Laboratory of Nasopharyngeal Carcinoma Diagnosis and Therapy, 651 Dongfeng Road East, Guangzhou 510060, China. \\ Email: hezhy@sysucc.org.cn.
}

Background: No consensus exists regarding the follow-up of nasopharyngeal carcinoma (NPC) patients stratified by different histological subtypes. The purpose of this study was to determine the hazard function of disease-related death and assess the prognostic effect of early and late disease-related death in NPC according to histological subtypes.

Methods: We included non-metastatic NPC patients between 2004 and 2014 using the Surveillance, Epidemiology and End-Results (SEER) program. Life-table methods, Kaplan-Meier methods, and a multivariate Cox regression model were used in the analysis.

Results: We identified 2,845 patients in this study including 1,218 (42.8\%), 849 (29.8\%), and $778(27.3 \%)$ patients with keratinizing squamous cell carcinoma (KSCC), differentiated non-keratinizing carcinoma (DNKC), and undifferentiated non-keratinizing carcinoma (UNKC), respectively. Most NPC-related death $(89.8 \%)$ occurred within 5 years of diagnosis. In the entire cohort, the hazard curve for NPC-related death peaked at 2 years. It peaked at 1 year, 2- and 5-year, and 2- and 6-year in patients with KSCC, DNKC, and UNKC, respectively. Within the follow-up period over 5 years, patients with DNKC had poorer NPCspecific survival (NPC-SS) compared to UNKC, and had comparable NPC-SS between the two subtypes after more than 5 years of follow-up. Moreover, within the follow-up period of 1, 2, and 3 years, patients with KSCC experienced poorer NPC-SS compared to UNKC but there was comparable NPC-SS between KSCC and UNKC patients after more than 3 years of follow-up.

Conclusions: The hazard rate patterns for NPC-related mortality significantly differed between histological subtypes. Tailored surveillance and follow-up strategies should be designed in NPC patients according to histological subtypes.

Keywords: Nasopharyngeal carcinoma (NPC); histological subtypes; keratinizing; non-keratinizing; survival

Submitted Aug 13, 2019. Accepted for publication Nov 01, 2019.

doi: $10.21037 /$ atm.2019.11.75

View this article at: http://dx.doi.org/10.21037/atm.2019.11.75 


\section{Introduction}

Although the incidence of nasopharyngeal carcinoma (NPC) accounted for approximately $0.7 \%$ of all new cancer cases diagnosed in 2018, a very unique pattern of geographic and ethnic distribution was observed (1). This cancer has the highest prevalence in the south of China (30 cases per 100,000 population) but the annual incidence of this cancer in Europe and the United States (US) is rare (less than 1 case per 100,000 population (1). In 2005, the World Health Organization (WHO) classified NPC into the following two histologic subtypes based on microscopic morphology: keratinizing squamous cell carcinoma (KSCC, WHO type I), and non-keratinizing carcinoma (NKC) (2). NKC subtype was further classed as being 'differentiated' (DNKC) and 'undifferentiated' (UNKC) (2). There are environmental, genetic, and ethnic factors linked to the development of the aforementioned histological categories (3-5). KSCC is the predominant histological type observed in Caucasians, whereas only $0.4-1 \%$ of these is identified in Chinese populations (6-11).

There have been limited studies comparing the survival of different histological subtypes from NPC endemic areas due to the low incidence of KSCC and DNKC in endemic areas $(10,12,13)$. Several studies from non-endemic areas found that the outcome of KSCC was significantly poorer than for NKC $(14,15)$ but further analysis of outcomes for DNKC and UNKC have not been performed. However, previous studies have found that those patients with a DNKC subtype had poor survival compared to those with UNKC subtype $(16,17)$. For the KSCC subtype, the disease failure pattern was mainly local recurrence, while distant metastasis was the most common reason for disease failure in those with NKC subtype $(18,19)$. A study from NPC endemic areas previously indicated that 2 years was the peak follow-up time for disease failure associated with NPC and most patients were the NKC subtype (20). However, to the best of our knowledge, no consensus exists regarding the follow-up of NPC patients stratified by different histological subtypes. Early diagnosis of disease recurrence could result in aggressive disease management with curative intent and improved outcome in selected patients. Therefore, the aims of this study were to determine the hazard function of disease-related death and determine the prognostic effect of early and late disease-related death in NPC according to histological subtypes. This may provide a tailored follow-up strategy for different histological subtypes using real-world data.

\section{Methods}

\section{Database and patients}

We employed retrospective data from the Surveillance, Epidemiology and End-Results (SEER) program in this study (21). The SEER program includes incidence, demographic, clinicopathological, treatment and outcome data for cancer in approximately $28 \%$ of the US population. Patients diagnosed with KSCC (ICD-O-3 codes 8070 and 8071), DNKC (ICD-O-3 codes 8072 and 8073), and UNKC (ICD-O-3 codes 8020, 8021, 8082, and 8083) types of NPC were identified based on the third edition of the International Classification of Diseases for Oncology (ICD-O-3). In addition, patients who received beam radiotherapy, available tumor $(\mathrm{T})$ stage, nodal $(\mathrm{N})$ stage were included, while race/ethnicity were also recorded. Patients with metastatic disease and no pathological diagnosis were excluded. The Institutional Review Board approval of this study was not required because the SEER database is a globally accessible database to the public.

\section{Variables}

The following variables were identified from the SEER database in this study: age, gender, histological subtypes, $\mathrm{T}$ stage, $\mathrm{N}$ stage, race/ethnicity, and chemotherapy. The definition of the staging system was based on the sixth edition of Union for International Cancer Control/ American Joint Committee on Cancer TNM classification system. The primary clinical end-point of the study was NPC-specific survival (NPC-SS).

\section{Statistical analysis}

Patient characteristics were compared using the Chisquared test. The life-table method was used to calculate the annual NPC-related death hazard rate over time i.e., the conditional probability of manifesting NPC relateddeath in a year given that the patient was clinically free from NPC related-death at the beginning of the year. KaplanMeier curves for NPC-SS were plotted and compared using the log-rank test. Univariate and multivariate Cox regression analyses were performed to investigate the independent prognostic indicators associated with NPCSS, and the results were presented as hazard ratios (HRs) and $95 \%$ confidence intervals (CIs). All statistical analyses were conducted in SPSS, version 22.0 (IBM Corporation, 
Table 1 Patient characteristics

\begin{tabular}{|c|c|}
\hline Variables & $\mathrm{n}$ \\
\hline \multicolumn{2}{|l|}{ Age (years) } \\
\hline$<65$ & 2,184 \\
\hline$\geq 65$ & 661 \\
\hline \multicolumn{2}{|l|}{ Gender } \\
\hline Male & 1,989 \\
\hline Female & 856 \\
\hline \multicolumn{2}{|l|}{ Race/ethnicity } \\
\hline Non-Hispanic White & 1,228 \\
\hline Non-Hispanic Black & 320 \\
\hline Hispanic (all races) & 232 \\
\hline Non-Hispanic Asian or Pacific Islander & 1,026 \\
\hline Non-Hispanic American Indian/Alaska Native & 39 \\
\hline \multicolumn{2}{|l|}{ Histology } \\
\hline Keratinizing squamous carcinoma & 1,218 \\
\hline Differentiated non-keratinizing carcinoma & 849 \\
\hline Undifferentiated non-keratinizing carcinoma & 778 \\
\hline \multicolumn{2}{|l|}{ Tumor stage } \\
\hline $\mathrm{T} 1$ & 886 \\
\hline $\mathrm{T} 2$ & 769 \\
\hline T3 & 576 \\
\hline $\mathrm{T} 4$ & 614 \\
\hline \multicolumn{2}{|l|}{ Nodal stage } \\
\hline NO & 735 \\
\hline N1 & 948 \\
\hline N2 & 845 \\
\hline N3 & 317 \\
\hline \multicolumn{2}{|l|}{ Chemotherapy } \\
\hline No/unknown & 346 \\
\hline Yes & 2,499 \\
\hline \multicolumn{2}{|l|}{ All cause of death } \\
\hline No & 1,819 \\
\hline Yes & 1,026 \\
\hline \multicolumn{2}{|l|}{ NPC-related death } \\
\hline No & 2,344 \\
\hline Yes & 501 \\
\hline
\end{tabular}

Armonk, NY, USA) and STATA version 14.0 (StataCorp., Texas, USA), and $\mathrm{P}$ values less than 0.05 were considered statistically significant.

\section{Results}

\section{Patient characteristics}

A total of 2,845 patients were identified in this study. Table 1 lists the patient characteristics in the entire cohort. A total of 249 (8.8\%), 751 (26.4\%), 957 (33.6\%), and 888 (31.2\%) patients were stage I, II, III, and IVA-B disease, respectively. Of these, 1,218 (42.8\%), 849 (29.8\%), and $778(27.3 \%)$ patients were defined as having KSCC, DNKC, and UNKC, respectively. There was comparable distribution of TNM stage among the three histological subtypes $(\mathrm{P}=0.191)$.

The median follow-up time was 45 months (range, 0-143 months), and 2,443 (85.9\%), 1,988 (69.9\%), 1,628 (57.2\%), 1,343 (47.2\%), 1,084 (38.1\%), and 847 (29.8\%) patients were followed up for a period of between 2 to 7 years, respectively. During follow-up, 1,819 deaths occurred including 501 NPC-related deaths. Most of these $(89.8 \%, \mathrm{n}=450)$ died within 5 years of their diagnosis.

\section{Hazard function}

In the entire cohort, the hazard curve of NPC-related death peaked at 2 years $(6 \%)$ but then slowly decreased over time, where the hazard rate was $1.0 \%$ during the 7 years of follow-up. We then performed subgroup analyses by different histological subtypes. Interestingly, the hazard curve showed specific patterns of NPC-related death according to various histological subtypes. In patients with KSCC, NPC-related deaths peaked at 1 year (9\%), and then slowly decreased over time, where the hazard rate was $1.0 \%$ during the 7 years of follow-up. In addition, there were two peaks in patients with DNKC; the first peak was within 2 years (7\%), and the second peak occurred at 5 years $(6 \%)$, before declining sharply. Moreover, in patients with UNKC, two peaks were also noted for NPC-related death; the first peak occurred at 2 years (4\%) and the second peak at 6 years (4\%). Figure 1 lists the hazard curve of NPC-related deaths in the entire cohort and by specific histological subtypes. 


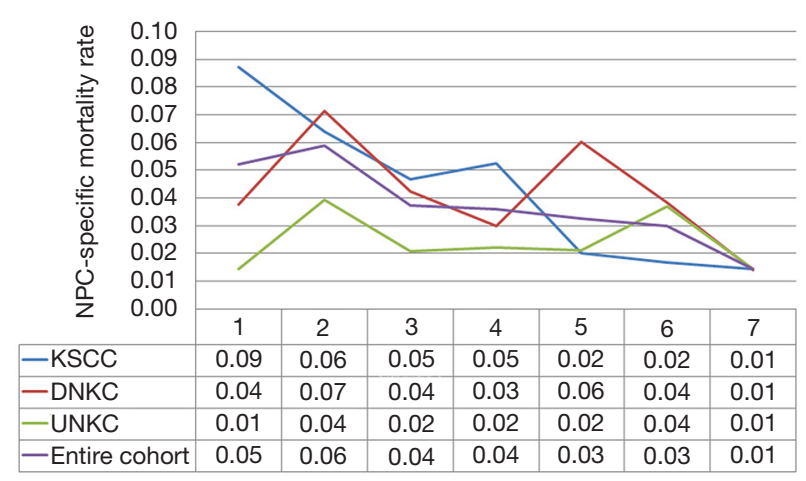

Figure 1 Annual hazard rates for NPC related-death by histological subtypes. NPC, nasopharyngeal carcinoma

\section{The effect of histological subtypes on outcome with extensive follow-up}

The results of univariate analysis are listed in Table 2. Within the follow-up time of 1, 2, 3, 4, and 5 years, patients with UNKC have better NPC-SS compared to DNKC. However, there were comparable NPC-SS between UNKC and DNKC after surviving more than 6 years. In addition, within the follow-up time of 1 year, patients with KSCC have lower NPC-SS compared to DNKC. However, there was comparable or non-inferior NPC-SS in KSCC subtype compared with DNKC after surviving more than 2 years.

Eight multivariate Cox regression models were performed to assess the impact of histological subtypes on outcome with prolonged follow-up (Table 3). The multivariate Cox regression analyses of NPC-SS were adjusted by age, race/ethnicity, $\mathrm{T}$ stage, $\mathrm{N}$ stage, and chemotherapy. For the entire cohort, the results showed that patients with KSCC had poorer NPC-SS than UNKC (HR 2.323, 95\% CI: 1.636-3.297, P<0.001), while NPC-SS was comparable between DNKC and UNKC (HR 1.435, 95\% CI: 0.945-2.111, $\mathrm{P}=0.067$ ). Within the follow-up time of $1,2,3,4$, and 5 years, patients with DNKC had poorer NPC-SS compared to UNKC but had comparable NPCSS between the two subtypes when 5 years of follow-up was exceeded. Moreover, within the follow-up period of 1, 2 and 3 years, patients with KSCC had poorer NPC-SS compared to UNKC but had comparable NPC-SS between KSCC and UNKC after more than 3 years of follow-up. The survival curves according to various histological subtypes on NPC-SS with prolonged follow-up are listed in Figure 2.

\section{Discussion}

In the current study, we used comprehensive real-world data to investigate the effect of histological subtypes on outcome in NPC patients after extensive follow-up. Our study suggests that there are significant differences in the patterns of hazard function according to histological subtypes. Patients with KSCC had comparable NPC-SS compared to UNKC after 3 years of follow-up, while DNKC showed a similar NPC-SS compared to UNKC, after 5 years of follow-up.

The understanding of the changes in the risk of NPCrelated death could help radiation oncologists to tailor more individualized surveillance and follow-up strategies of NPC patients. For the entire cohort, our study showed that the hazard curve for NPC-related death peaked at 2 years, and then declined gradually between 3 and 6 years. The study from Sun Yat-sen University Cancer Center included 749 patients with NPC (99.3\% with NKC, and $0.7 \%$ with KSCC) also indicated that the hazard curve for treatment failure peaked at 2 years, and between 3 and 5 years it slowly declined and then dropped sharply (20). This was similar to our findings. However, the aforementioned results did not distinguish by outcome between DNKC and UNKC (20). In our study, we included NPC patients from non-epidemic areas where KSCC is the predominant histology type. Previous studies have shown differences in the risk of disease failure between DNKC and UNKC $(16,17)$. To the best of our knowledge, no consensus exists regarding the follow-up of NPC patients in assessing the risk of NPC-related deaths over time by different histological subtypes. Our subgroup analysis indicated that the risk of NPC-related death for those with a KSCC subtype was $9 \%$ within 1 year and $2.0 \%$ during the first 5 years of follow-up. In addition, both DNKC and UNKC had two peaks for NPC-related death.

For patients with KSCC, the predominant pattern of disease failure was local recurrence, while for $\mathrm{NKC}$, distant recurrence was the predominant pattern $(18,19)$. Therefore, the higher risk of mortality associated with KSCC may contribute to uncontrolled locoregional tumors in the first year of diagnosis. Although the majority of NPC-related deaths occurred within 2 years of diagnosis, the findings from our study indicate that the hazard rate was $6.0 \%$ in DNKC patients who developed a late NPCrelated death during the 5 years of follow-up, while UNKC 
Table 2 Univariate Cox regression analyses on outcome with prolonged follow-up

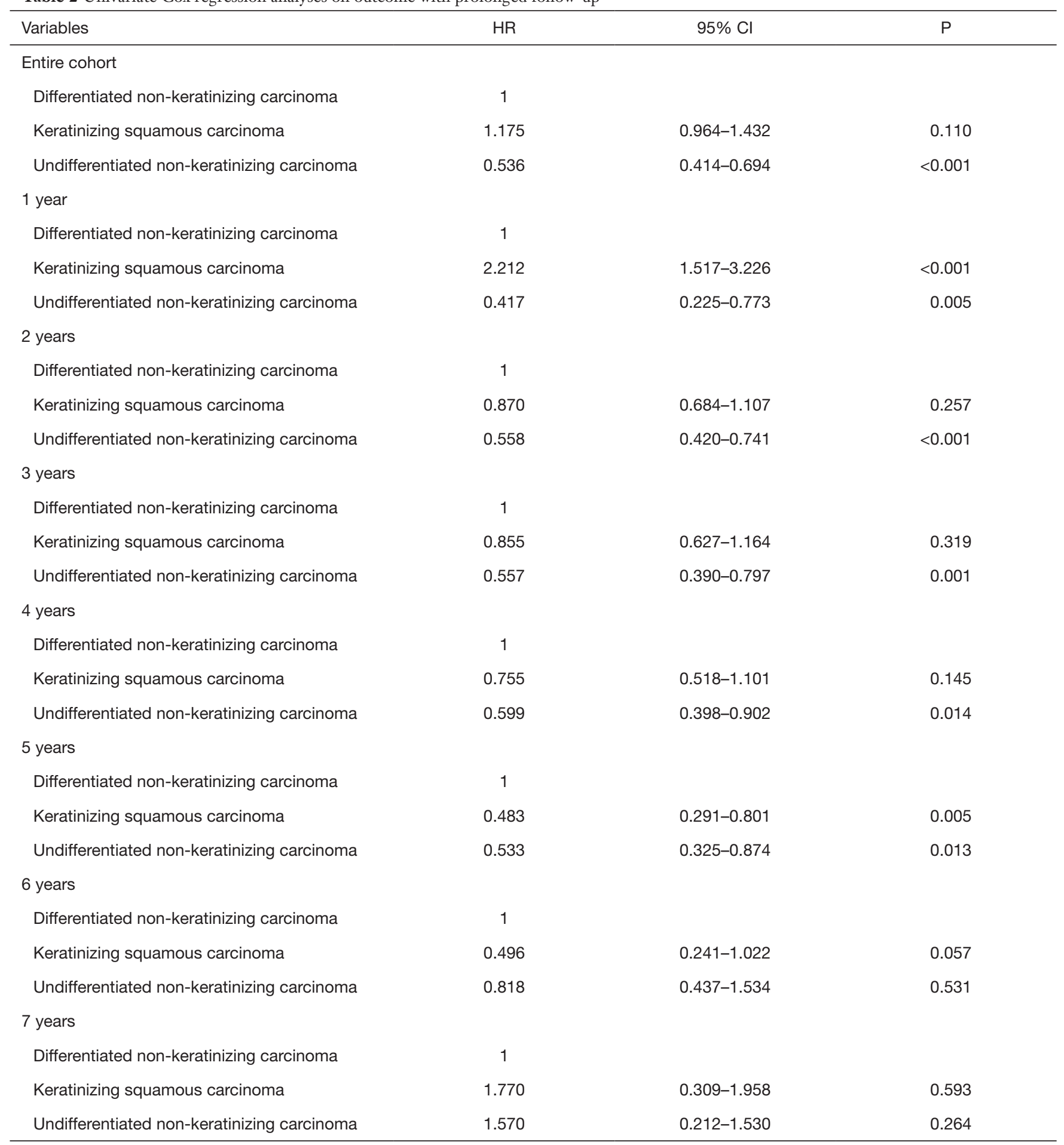


Table 3 Multivariate Cox regression analyses on outcome with prolonged follow-up

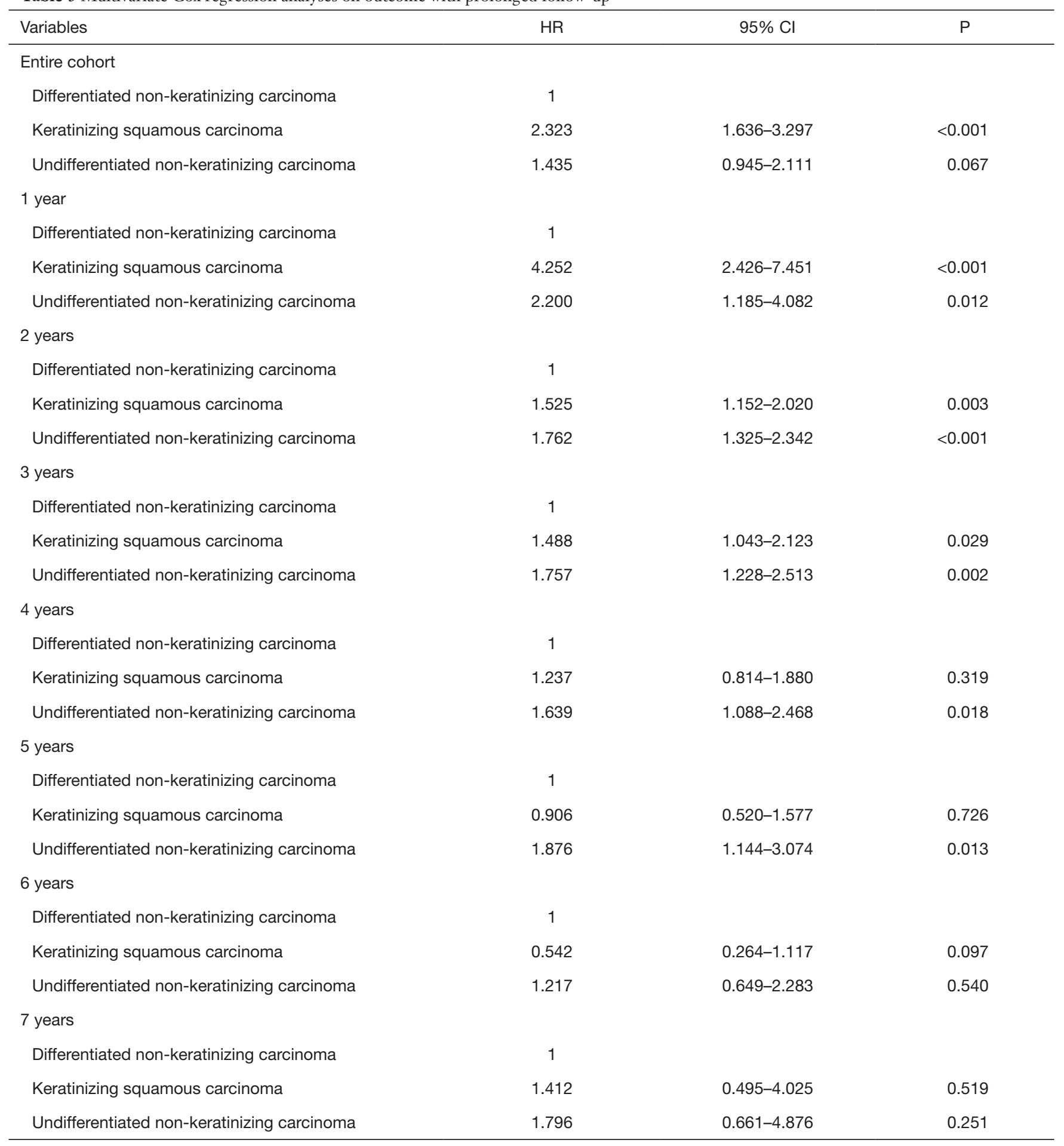




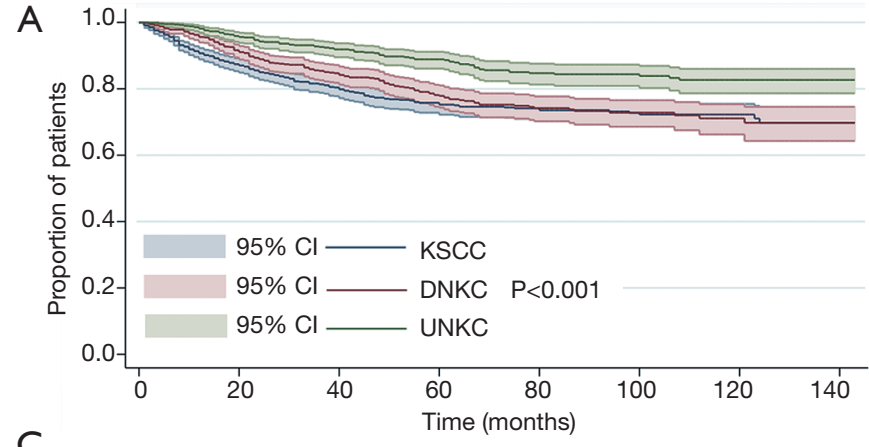

C

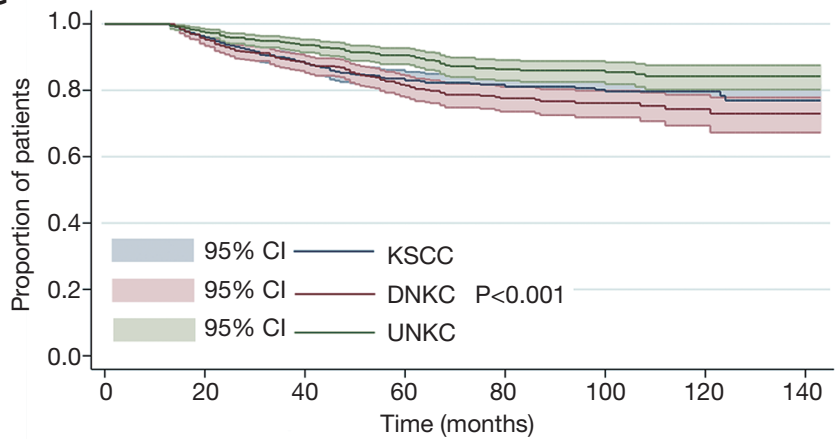

E

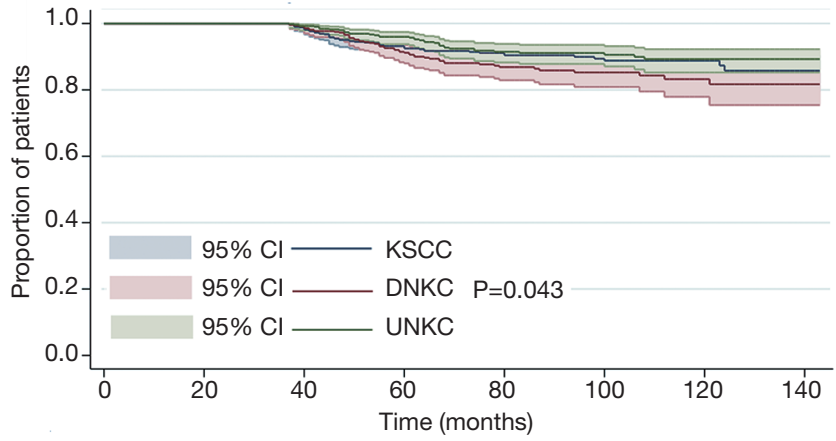

G

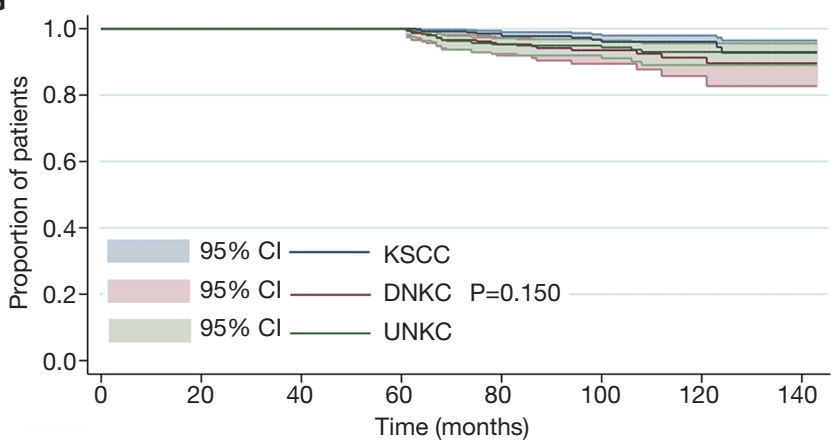

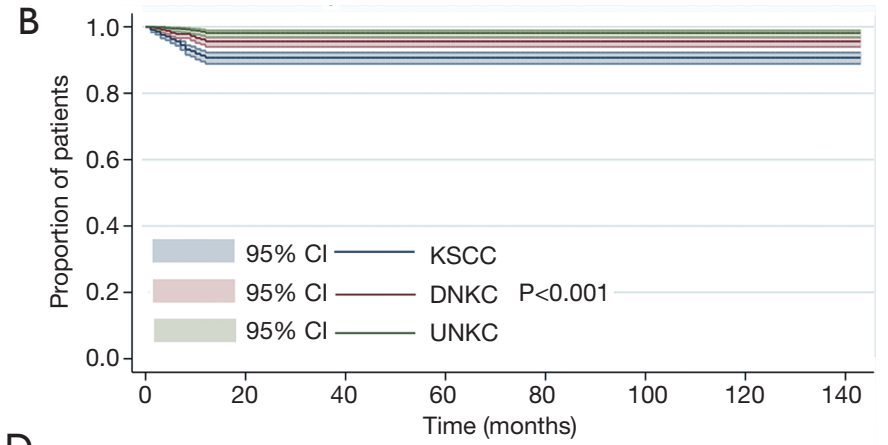

D

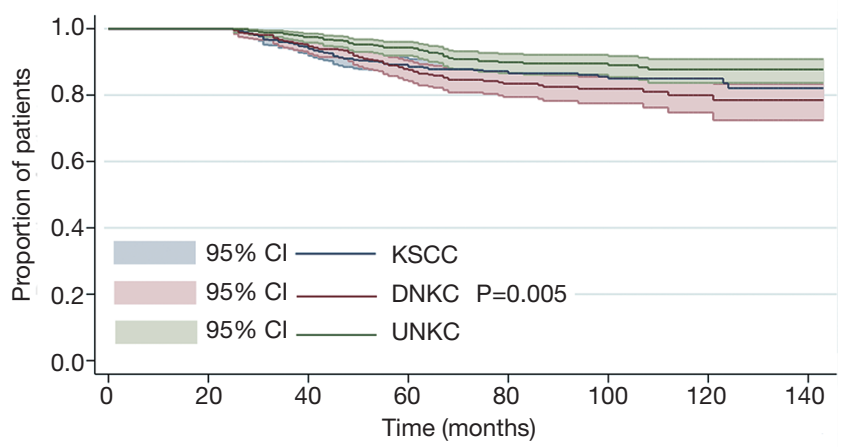

$\mathrm{F}$

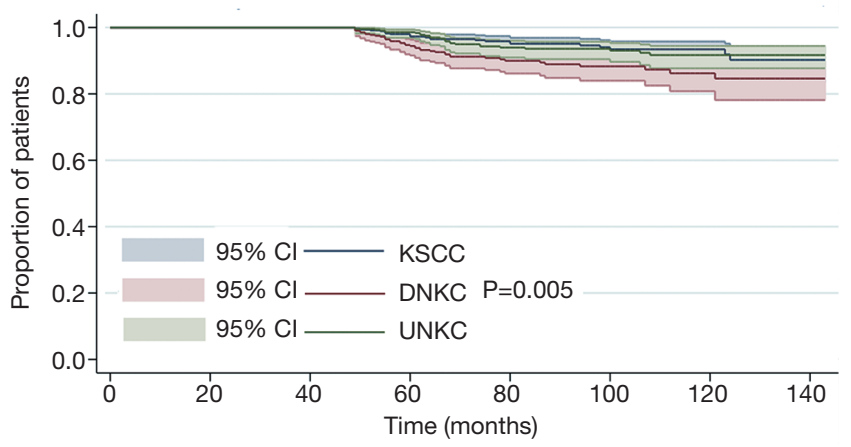

$\mathrm{H}$

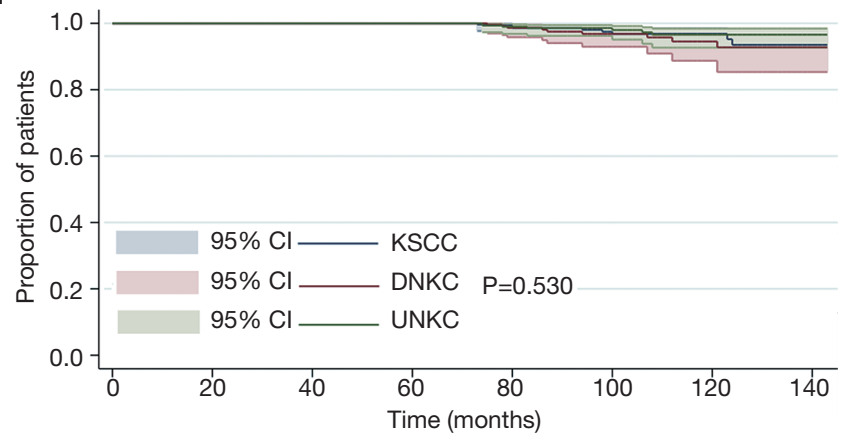

Figure 2 The survival curves according to various histological subtypes of nasopharyngeal carcinoma-specific survival with prolonged follow-up (A, entire cohort; B, follow-up of 1 year; C, follow-up of 2 years; D, follow-up of 3 years; E, follow-up of 4 years; F, follow-up of 5 years; G, follow-up of 6 years; H, follow-up of 7 years). 
patients developed a late NPC-related death during the 6 years of follow-up. The tumor dormancy hypothesis includes immunosurveillance, angiogenic dormancy, and cellular dormancy (G0-G1 arrest), and these may be the mechanisms that explain this phenomenon (22). Several compelling preclinical studies have indicated that angiogenesis switch may be a key trigger in reversing tumor dormancy, which could cause tumor local recurrence and distant metastasis $(23,24)$. These findings suggest that a deeper understanding of the underlying tumor biology that drives dormancy in NPC patients is needed in order to better elucidate by different histological subtypes.

The different response mechanisms to the same treatment may also be one of the biological mechanisms responsible for different risks in NPC-related death by histological subtypes. In a study of patients from a NPC epidemic area, the expression of drug resistance-related protein-excision repair cross complementation group 1 (ERCC1) was significantly higher in the DNKC subtype compared with the UNKC subtype. Patients with ERCC1 overexpression had a significantly higher risk of locoregional and distant failure than patients with low ERCC1 expression (16). However, no patients had KSCC in the aforementioned study. Previously, it has been reported that low ERCC1 expression was related to better survival benefit from cisplatin-based chemotherapy in non-small-cell lung cancer (25). In current clinical practice, cisplatin-based chemotherapy was also the main chemotherapy regimen for NPC. A similar observation of poor response to treatment and outcome with overexpression of ERCC1 in KSCC or DNKC subtypes compared to the UNKC subtype has been reported from patients in a non-endemic region (17). Therefore, patients with ERCC1 overexpression may have a lower response to radiotherapy. Given the different radiosensitivities of various histological subtypes, we believe that the relatively higher proportion of the KSCC and DNKC subtypes is the main reason for poorer survival in non-endemic areas (7).

The current follow-up guideline for NPC from the National Comprehensive Cancer Network recommends follow-up every $1-3,2-6$, and 4-8 months in years 1,2 , and $3-5$, respectively (26). Our study queries whether it is reasonable to use the same intensive follow-up strategy in NPC patients with different histological subtypes. According to our results, strict follow-up should be performed in patients with KSCC diagnosed within 1-3 years compared with those who have UNKC, whereas patients with DNKC should strictly be followed up for at least 5 years. In patients with DNKC who are more likely to relapse, intensive monitoring the trend of changes in post treatment Epstein-Barr virus (EBV) DNA copies are critical. In addition, those who are at higher risk of relapse can be considered for investigational adjuvant treatment, for instance there is suggestion that tegafur may reduce risk of relapse $(27,28)$. Moreover, the other area worth looking into in the future is 3 and 12 months post treatment positron emission tomography-computer tomography in high risk subgroups, especially for KSCC to predict early relapse.

There were several limitations to our study. First, we could not include the patient characteristics such as EBV status, smoking status, alcohol consumption, socioeconomic environment, performance status, or comorbidities due to the retrospective nature of our study. This might have importance for assessing outcome of NPC, especially for KSCC subtypes (29). Secondly, the SEER database also lacks details regarding chemotherapy regimen, radiotherapy technique, radiotherapy dose, completion rate of therapy, and sequence of chemotherapy and radiotherapy. Therefore, treatment strategies were somewhat heterogenous in our study. Moreover, we only analyzed NPC-SS in this study due to lack of patterns of locoregional and distant recurrence. Finally, our findings may not be representative of populations from NPC epidemic areas. However, we believe our study is the first study to assess the hazard rate of NPC-related mortality according to histological subtypes using real-world data.

\section{Conclusions}

In conclusion, our study suggests that the patterns of hazard rates for NPC-related mortality significantly differed among patients with different histological subtypes. Tailored surveillance and follow-up strategies should be devised for NPC patients according to their histological subtype.

\section{Acknowledgments}

The authors acknowledge the efforts of the Surveillance, Epidemiology, and End Results (SEER) Program tumor registries in the creation of the SEER database.

\section{Footnote}

Conflicts of Interest: The authors have no conflicts of interest 
to declare.

Ethical Statement: The authors are accountable for all aspects of the work in ensuring that questions related to the accuracy or integrity of any part of the work are appropriately investigated and resolved. The approval process of Institutional Review Board was waived because of the de-identified information of the patients included in the SEER.

\section{References}

1. Bray F, Ferlay J, Soerjomataram I, et al. Global cancer statistics 2018: GLOBOCAN estimates of incidence and mortality worldwide for 36 cancers in 185 countries. CA Cancer J Clin 2018;68:394-424.

2. Barnes L, Eveson JW, Reichart P, et al. World Health Organization Classification of Tumours: Pathology and Genetics of Head and Neck Tumours. Lyon, France: IARC Press, 2005.

3. Chan JKC, Bray F, Mc Carron P, et al. Nasopharyngeal carcinoma. In: Barnes EL, Eveson JW, Reichart P, Sdransky D, editors. Pathology and genetics of head and neck tumours. Kleihues P, Sobin LH. editors. World Health Organization Classification of Tumours. Lyon, France: IARC Press, 2005:85-97.

4. Yu WM, Hussain SS. Incidence of nasopharyngeal carcinoma in Chinese immigrants, compared with Chinese in China and South East Asia: review. J Laryngol Otol 2009;123:1067-74.

5. Roy Chattopadhyay N, Das P, Chatterjee K, et al. Higher incidence of nasopharyngeal carcinoma in some regions in the world confers for interplay between genetic factors and external stimuli. Drug Discov Ther 2017;11:170-80.

6. Ou SH, Zell JA, Ziogas A, et al. Epidemiology of nasopharyngeal carcinoma in the United States: improved survival of Chinese patients within the keratinizing squamous cell carcinoma histology. Ann Oncol 2007;18:29-35.

7. Ruuskanen M, Grenman R, Leivo I, et al. Outcome of nasopharyngeal carcinoma in Finland: A nationwide study. Acta Oncol 2018;57:251-6.

8. Arnold M, Wildeman MA, Visser O, et al. Lower mortality from nasopharyngeal cancer in The Netherlands since 1970 with differential incidence trends in histopathology. Oral Oncol 2013;49:237-43.

9. Tang LL, Chen YP, Mao YP, et al. Validation of the 8th Edition of the UICC/AJCC Staging System for
Nasopharyngeal Carcinoma From Endemic Areas in the Intensity-Modulated Radiotherapy Era. J Natl Compr Canc Netw 2017;15:913-9.

10. Pan JJ, Ng WT, Zong JF, et al. Proposal for the 8th edition of the AJCC/UICC staging system for nasopharyngeal cancer in the era of intensity-modulated radiotherapy. Cancer 2016;122:546-58.

11. Lee AW, Sze WM, Au JS, et al. Treatment results for nasopharyngeal carcinoma in the modern era: the Hong Kong experience. Int J Radiat Oncol Biol Phys 2005;61:1107-16.

12. Chan AT, Teo ML, Lee WY, et al. The significance of keratinizing squamous cell histology in Chinese patients with nasopharyngeal carcinoma. Clin Oncol (R Coll Radiol) 1998;10:161-4.

13. Kurniawan AN, Susworo R, Sumanto. Nasopharyngeal carcinoma: correlation of histopathology with radiation response. Southeast Asian J Trop Med Public Health 1985;16:613-8.

14. Vazquez A, Khan MN, Govindaraj S, et al. Nasopharyngeal squamous cell carcinoma: a comparative analysis of keratinizing and nonkeratinizing subtypes. Int Forum Allergy Rhinol 2014;4:675-83.

15. García-Lorenzo J, Farre N, Codina A, et al. Nasopharyngeal carcinoma: 30-year experience of a single institution in a non-endemic area. Clin Transl Oncol 2017;19:777-83.

16. Cheung F, Chan O, Ng WT, et al. The prognostic value of histological typing in nasopharyngeal carcinoma. Oral Oncol 2012;48:429-33.

17. Lee HW, Hwang YH, Han JH, et al. High expression of excision repair cross-complementation group 1 protein predicts poor outcome in patients with nasopharyngeal cancer. Oral Oncol 2010;46:209-13.

18. Colaco RJ, Betts G, Donne A, et al. Nasopharyngeal carcinoma: a retrospective review of demographics, treatment and patient outcome in a single centre. Clin Oncol (R Coll Radiol) 2013;25:171-7.

19. Reddy SP, Raslan WF, Gooneratne S, et al. Prognostic significance of keratinization in nasopharyngeal carcinoma. Am J Otolaryngol 1995;16:103-8.

20. Liu X, Tang LL, Du XJ, et al. Changes in Disease Failure Risk of Nasopharyngeal Carcinoma over Time: Analysis of 749 Patients with Long-Term Follow-Up. J Cancer 2017;8:455-9.

21. Surveillance, Epidemiology, and End Results (SEER) Program (www.seer.cancer.gov) SEER ${ }^{*}$ Stat Database: Incidence - SEER 18 Regs Research Data + Hurricane 
Katrina Impacted Louisiana Cases, Nov 2016 Sub (19732014 varying) - Linked To County Attributes - Total U.S., 1969-2015 Counties, National Cancer Institute, DCCPS, Surveillance Research Program, Surveillance Systems Branch, released April 2017, based on the November 2016 submission.

22. Aguirre-Ghiso JA. Models, mechanisms and clinical evidence for cancer dormancy. Nat Rev Cancer 2007;7:834-46.

23. Almog N, Henke V, Flores L, et al. Prolonged dormancy of human liposarcoma is associated with impaired tumor angiogenesis. FASEB J 2006;20:947-9.

24. Bergers G, Benjamin LE. Tumorigenesis and the angiogenic switch. Nat Rev Cancer 2003;3:401-10.

25. Olaussen KA, Dunant A, Fouret P, et al. DNA repair by ERCC1 in non-small-cell lung cancer and cisplatin-

Cite this article as: Wu SG, Lian CL, Wang J, Zhang WW, Sun JY, Lin Q, He ZY. The effect of histological subtypes on survival outcome in nasopharyngeal carcinoma after extensive follow up. Ann Transl Med 2019;7(23):768. doi: 10.21037/ atm.2019.11.75 based adjuvant chemotherapy. N Engl J Med 2006;355:983-91.

26. National Comprehensive Cancer Network. Available online: https://www.nccn.org/professionals/physician_gls/ pdf/head-and-neck.pdf [access, MAR 25, 2019].

27. Chen JH, Huang WY, Ho CL, et al. Evaluation of oral tegafur-uracil as metronomic therapy following concurrent chemoradiotherapy in patients with non-distant metastatic TNM stage IV nasopharyngeal carcinoma. Head Neck 2019;41:3775-82.

28. Liu YC, Wang WY, Twu CW, et al. Prognostic impact of adjuvant chemotherapy in high-risk nasopharyngeal carcinoma patients. Oral Oncol 2017;64:15-21.

29. Ji X, Zhang W, Xie C, et al. Nasopharyngeal carcinoma risk by histologic type in central China: impact of smoking, alcohol and family history. Int J Cancer 2011;129:724-32. 